\title{
THE RELATIONSHIPS BETWEEN COMPRESSIVE STRENGTH AND DENSITY OF POLYSTYRENE LIGHTWEIGHT CONCRETE AND THEIR COMPONENT RATIOS
}

\section{Scopus

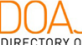

Mohammed Salman Al-lami

Al-Isra University,

Faculty of Engineering,

Departement of Civil

Engeneering,

Amman, Jordan

\section{Emad Abdul-Majeed Al-saadi}

Al-Zawia University,

Faculty of Engineering,

Departement of Civil

Engeneering,

Sabratah, Lybia

Key words: lightweight concrete, compressive strength, density, polystyrene, sand, cement, mix proportions

\section{Cite article:}

Mohammed, S. A., \& Emad, A. A. [2021]. The relationships between compressive strength and density of polystyrene lightweight concrete and their component ratios. Journal of Applied Engineering Science, 19(1), 175 - 185. DOI:10.5937/jaes0-27471

Online aceess of full paper is available at: www.engineeringscience.rs/browse-issues 


\title{
THE RELATIONSHIPS BETWEEN COMPRESSIVE STRENGTH AND DENSITY OF POLYSTYRENE LIGHTWEIGHT CONCRETE AND THEIR COMPONENT RATIOS
}

\author{
Mohammed Salman Al-lami ${ }^{*}$, Emad Abdul-Majeed Al-saadi ${ }^{2}$ \\ ${ }^{1}$ Al-Isra University, Faculty of Engineering, Departement of Civil Engeneering, Amman, Jordan \\ ${ }^{2}$ Al-Zawia University, Faculty of Engineering, Departement of Civil Engeneering, Sabratah, Lybia
}

The research deals with the compressive strength and density of lightweight concrete, made from polystyrene, cement, sand and water. This type of lightweight concrete is characterized by good thermal insulation, low density and good compressive strength and it is used as a lining material in inclination surfaces and in the production of unloaded building units and elements. The aim of this paper is to suggest mathematical relationships between compressive strength, density and mix proportions. An intensive experimental program has been implemented for this purpose. The investigated variables were the mix proportions, namely, polystyrene to cement ratio $(P / C)$, sand to cement ratio $(S / C)$ and water to cement ratio (W/C). Sixty mixes were produced and tested using five polystyrene to cement ratios $(P / C), 0.02,0.03,0.04,0.05,0.06$ four sand to cement ratios $(S / C), 2.5,3,3.5,4$, and three water to cement ratios (W/C), 0.35, 0.40, 0.45. From the test results, mathematical relationships were suggested that can be of use in determining mix proportions of polystyrene lightweight concrete based on the required compressive strength or based on the required dry density.

Key words: lightweight concrete, compressive strength, density, polystyrene, sand, cement, mix proportions

\section{INTRODUCTION}

Lightweight concrete is considered one of the alternatives that has a great importance in modern buildings. It is one of the most prominent developments in concrete technology and has a wide use in the construction works because of its characteristics that traditional concrete does not provide. It is more economical because of its low density, ranging between $(240-1840) \mathrm{kg} / \mathrm{m}^{3}$ [1] and [2], reducing dead loads and resulting in smaller structural elements. In addition, polystyrene is available locally with low cost, and its waste can be used in abundance, which contributes to reducing environmental pollution. Also, it provides a good thermal insulation, saving the energy needed for cooling and heating, especially, the polystyrene is classified as first-class thermal insulation material. Kohling [3] conducted a research and showed the possibility of using polystyrene as aggregate to manufacture concrete with high thermal insulation. Maura [4] found that the compression strength of polystyrene concrete with densities ranging between $(220-460) \mathrm{kg} / \mathrm{m}^{3}$ is in the range of $(0.7-2.3) \mathrm{Mpa}$, while the modulus of rupture ranges between Mpa $(0.3-0.36)$. Parton and barbary [5] observed that the properties of polystyrene concrete such as density, compressive strength, modulus of rupture, and thermal insulation mainly depend on the Polystyrene to cement ratio. Sabaa and Ravindrarajah [6] carried out the experimental program using the expanded polystyrene waste in a granular form as lightweight aggregate to produce lightweight structural concrete with the unit weight varying from 1600 to $2000 \mathrm{~kg} / \mathrm{m}^{3}$. The coarse aggregate replacements levels used were 30 , 50 and $70 \%$. The results showed that drying shrinkage and creep of polystyrene aggregate concrete increased, whereas compressive strength and modulus of elasticity decreased with decrease in the density of concrete. The compressive strength was found to be more sensitive to the density than the modulus of elasticity. The ultimate creep coefficient for polystyrene aggregate concrete in the density range of 1600 to $2000 \mathrm{~kg} / \mathrm{m}^{3}$ was between 1.90 and 2.37 for the concrete with the cement content of $410 \mathrm{~kg} / \mathrm{m}^{3}$. Kuhail and Shihada [7] suggested a recommended way in preparing lightweight polystyrene concrete mixes and proportions for producing homogeneous mixes with compressive strengths ranging between 17 $\mathrm{MPa}$ to $25 \mathrm{MPa}$ and densities ranging from $1650 \mathrm{~kg} / \mathrm{m}^{3}$ to $1830 \mathrm{~kg} / \mathrm{m}^{3}$. Kan and Demirbogab [8] observed that, with the increase of cement to EPS beads ratio in the mixes, both density and compressive strength of concretes increased up to 4 and 350 times respectively, when compared to the control sample. Hardened concrete densities and compressive strength varied from 464 to 1940 $\mathrm{kg} / \mathrm{m}^{3}$, and 0.11 to $38.50 \mathrm{MPa}$, respectively. Slump values of the mixes were changed from 8 to $43 \mathrm{~mm}$. Herki et al. [9] used waste polystyrene as a lightweight aggregate (LWA) to produce lightweight aggregate concrete (LWAC). In this study the WP was shredded to different sizes. Four concrete mixtures in total with varying WP content ratios of $0,20,40$ and $60 \%$ as partial replacement of natural coarse aggregate by equivalent volume was prepared. The water/cement (W/C) ratio of 0.5 was kept constant for all concrete mixtures. Mechanical and durability properties including compressive strength, water absorption (WA) and density was determined for the various concrete mixtures at the ages of 7 and 28 days of 
water curing. According to the results obtained, the utilization of WP in concrete production is possible. Abd et al. [10] carried out an investigation to study the properties, such as compressive strength and tensile strengths of light weight concrete containing Expanded Polystyrene (EPS) beads. The results showed that the amount of polystyrene beads incorporated in concrete influences the properties of hardened concrete. Since the compressive strength less than a certain percentage depending on the amount of replacement. At 28 days, it was found that compressive strength of $5 \%, 15 \%, 20 \%$, EPS based concretes compared to control concrete were $41 \%$, $38 \%$, $25 \%$, respectively. Minh and Phuong et al [11] investigated the effect of properties and volume of the matrix on the properties of lightweight concrete. The results show that properties of structural polystyrene concrete, such as workability and compressive strength, depend on the aggregate size of the matrix. It also shows that decreasing aggregate size of the matrix is the effective way to increase workability and compressive strength of lightweight concrete. Bedekovic et al. [12] showed based on their test results on waste expanded polystyrene lightweight concrete a dependence of the component ratio on the bulk density ranging from $360 \mathrm{~kg} / \mathrm{m}^{3}$ to $915 \mathrm{~kg} / \mathrm{m}^{3}$ and compressive strength ranging from $0.385 \mathrm{MPa}$ to $2.538 \mathrm{MPa}$.

From the previous studies, it is clear that the researches dealing with the mix design of polystyrene lightweight concrete are very limited. Kuhail and Shihada [7] suggested a procedure for mix design based on the required compressive strength and density. This paper represents a contribution in this field by suggesting number of mathematical relationships between compressive strength and density of polystyrene lightweight concrete and their mix proportions namely, sand to cement ratio (S/C), water to cement ratio (W/C) and polystyrene to cement ratio $(\mathrm{P} / \mathrm{C})$, which can be of use to suggest the mix proportions based on the required compressive strength or based on the required dry density. Ubi, Stanley et al. [13] carried an experimental program to develop a mathematical model that will optimize the compressive strength of polystyrene lightweight concrete. The materials used for this study were, ordinary Portland cement, Water, Sand, coarse aggregate and Expanded Polystyrene beads. The study adopted the Scheffe's simplex lattice design for both pseudo and component proportion models to generate their respective mixes. The study showed that using the Scheffe's Pseudo component model, an optimized compressive strength value of $27.920 \mathrm{~N} / \mathrm{mm}^{2}$ can be obtained from a water, cement, sand and coarse aggregate (at $12 \%$ partial replacement with polystyrene aggregates) mix ratio of $0.455,1,1.820$ and 2.980 respectively

\section{EXPERIMENTAL PROGRAM}

\section{Mix proportions}

An experimental program was implemented including several laboratory tests on compressive strength and dry density of lightweight concrete using sand and polystyrene as aggregate. The investigated variables were the mix ratios of their components. These mixes were accomplished by using three water to cement ratios (W/C) of $0.35,0.40$ and 0.45 , five polystyrene to cement ratios (P/C), 0.02, 0.03, 0.04, 0.05, and 0.06, four sand to cement ratios, 2.5, 3, 3.5 and 4 . As a result, sixty mixes were produced and tested. Material weights were calculated per cubic meter using the volumetric equation to be as shown in Table 1.

Table 1: Detail of mixes

\begin{tabular}{|c|c|c|c|c|c|c|c|c|c|c|c|}
\hline $\begin{array}{l}\text { Mix } \\
\text { No. }\end{array}$ & P/C & $\begin{array}{l}\text { Water } \\
\mathrm{kg} / \mathrm{m}^{3}\end{array}$ & $\begin{array}{c}\text { Polystyrene } \\
\mathrm{kg} / \mathrm{m}^{3}\end{array}$ & $\begin{array}{l}\text { Sand } \\
\mathrm{kg} / \mathrm{m}^{3}\end{array}$ & $\begin{array}{c}\text { Cement } \\
\mathrm{kg} / \mathrm{m}^{3}\end{array}$ & $\begin{array}{l}\text { Mix } \\
\text { No. }\end{array}$ & $\mathrm{P} / \mathrm{C}$ & $\begin{array}{l}\text { Water } \\
\mathrm{kg} / \mathrm{m}^{3}\end{array}$ & \begin{tabular}{|c|} 
Polystyrene \\
$\mathbf{k g} / \mathrm{m}^{3}$
\end{tabular} & $\begin{array}{l}\text { Sand } \\
\mathrm{kg} / \mathrm{m}^{3}\end{array}$ & $\begin{array}{c}\text { Cement } \\
\mathrm{kg} / \mathrm{m}^{3}\end{array}$ \\
\hline \multicolumn{6}{|c|}{$\mathrm{W} / \mathrm{C}=0.35 \mathrm{~S} / \mathrm{C}=2.5$} & \multicolumn{6}{|c|}{$\mathrm{W} / \mathrm{C}=0.35 \mathrm{~S} / \mathrm{C}=3$} \\
\hline 2.1 .2 & 0.02 & 107.36 & 6.135 & 766.86 & 306.74 & 1.2 & 0.02 & 01.59 & 5.805 & 870.77 & 290.26 \\
\hline 2.1 .3 & 0.03 & 85.50 & 29 & 610.74 & .30 & 1.3 & 0.03 & .80 & & 701.17 & 3.72 \\
\hline 2.1 .4 & 0.04 & 71.04 & 8.119 & 507.44 & 202.98 & 3.1 .4 & 0.04 & 68.47 & 7.825 & 586.87 & 195.62 \\
\hline 2.1 .5 & 0.05 & 60.76 & 680 & 434.02 & 173.61 & 3.1 .5 & 0.05 & 58.87 & 8.410 & 504.61 & 168.20 \\
\hline 2.1 .6 & 0.06 & 53.08 & 9.100 & 379.17 & 67 & 6 & 0.06 & 51.63 & 0.051 & 442.57 & .52 \\
\hline \multicolumn{6}{|c|}{$\mathrm{W} / \mathrm{C}=0.40 \mathrm{~S} / \mathrm{C}=2.5$} & \multicolumn{6}{|c|}{$\mathrm{W} / \mathrm{C}=0.40 \mathrm{~S} / \mathrm{C}=3$} \\
\hline 2.2 .2 & 0.02 & 120.84 & 6.042 & 755.28 & 302.11 & 3.2 .2 & 0.02 & 114.44 & 5.722 & 858.31 & 286.10 \\
\hline 2.2 .3 & 0.03 & 96.54 & 40 & 603.37 & 241.35 & 3.2 .3 & 0.03 & 92.41 & 1 & 693.07 & 1.02 \\
\hline 2.2 .4 & 0.04 & 80.37 & 7 & 502.34 & 200.94 & 3.2 .4 & 0.04 & 77.49 & 7.749 & 581.18 & 3.73 \\
\hline 2.2 .5 & 0.05 & 68.85 & 8.606 & 430.29 & 172.12 & 3.2 .5 & 0.05 & 66.72 & 40 & 500.40 & 6.80 \\
\hline 2.2 .6 & 0.06 & 60.21 & 9.032 & 376.31 & 150.53 & 3.2 .6 & 0.06 & 58.58 & 8.787 & 439.33 & 3.44 \\
\hline \multicolumn{6}{|c|}{$\mathrm{W} / \mathrm{C}=0.45 \mathrm{~S} / \mathrm{C}=2.5$} & \multicolumn{6}{|c|}{$\mathrm{W} / \mathrm{C}=0.45 \mathrm{~S} / \mathrm{C}=3$} \\
\hline 2.3 .2 & 0.02 & 133.93 & 5.952 & 744.04 & 297.61 & 3.3 .2 & 0.02 & 127.29 & 5.689 & 845.85 & 281.94 \\
\hline 2.3 .3 & 0.03 & 107.31 & 7.154 & 596.18 & 238.47 & 3.3 .3 & 0.03 & 103.02 & 6.850 & 684.97 & 228.32 \\
\hline 2.3 .4 & 0.04 & 89.52 & 7.957 & 497.34 & 198.94 & 3.3 .4 & 0.04 & 86.51 & 7.673 & 575.49 & 191.84 \\
\hline 2.3 .5 & 0.05 & 76.79 & 8.532 & 426.62 & 170.65 & 3.3 .5 & 0.05 & 74.57 & 8.270 & 496.19 & 165.4 \\
\hline 2.3 .6 & 0.06 & 67.23 & 8.964 & 373.50 & 149.40 & 3.3.6 & 0.06 & 65.53 & 8.723 & 436.09 & 145.36 \\
\hline
\end{tabular}




\begin{tabular}{|c|c|c|c|c|c|c|c|c|c|c|c|}
\hline $\begin{array}{l}\text { Mix } \\
\text { No. }\end{array}$ & $\mathrm{P} / \mathrm{C}$ & $\begin{array}{l}\text { Water } \\
\mathrm{kg} / \mathrm{m}^{3}\end{array}$ & $\begin{array}{l}\text { Polysty- } \\
\text { rene } \mathrm{kg} / \mathrm{m}^{3}\end{array}$ & $\begin{array}{l}\text { Sand } \\
\mathrm{kg} / \mathrm{m}^{3}\end{array}$ & $\begin{array}{c}\text { Cement } \\
\mathrm{kg} / \mathrm{m}^{3}\end{array}$ & $\begin{array}{l}\text { Mix } \\
\text { No. }\end{array}$ & $\mathrm{P} / \mathrm{C}$ & $\begin{array}{l}\text { Water } \\
\mathrm{kg} / \mathrm{m}^{3}\end{array}$ & $\begin{array}{l}\text { Polysty- } \\
\text { rene } \mathrm{kg} / \mathrm{m}^{3}\end{array}$ & $\begin{array}{l}\text { Sand } \\
\mathrm{kg} / \mathrm{m}^{3}\end{array}$ & $\begin{array}{l}\text { Cement } \\
\mathrm{kg} / \mathrm{m}^{3}\end{array}$ \\
\hline \multicolumn{6}{|c|}{$\mathrm{W} / \mathrm{C}=0.35 \mathrm{~S} / \mathrm{C}=3.5$} & \multicolumn{6}{|c|}{$\mathrm{W} / \mathrm{C}=0.35 \mathrm{~S} / \mathrm{C}=4$} \\
\hline 4.1 .2 & .02 & 96.41 & 5.509 & 964.08 & 7545 & 12 & .02 & 91.73 & 5.242 & 1048.33 & 2.08 \\
\hline 4.1 .3 & 03 & 41 & 1 & 4.09 & & & .03 & 5.29 & 3 & 41 & \\
\hline 4.1 .4 & 0.04 & .07 & 1 & 74 & & & .04 & 342 & & 62 & \\
\hline 4.1 .5 & 0.0 & .09 & & & & & 5 & 5.42 & & 35 & 34 \\
\hline 4.1 .6 & .06 & 50.26 & 8.616 & 502.60 & & & 0.06 & 48.95 & & & \\
\hline \multicolumn{6}{|c|}{$\mathrm{W} / \mathrm{C}=0.40 \mathrm{~S} / \mathrm{C}=3.5$} & \multicolumn{6}{|c|}{$\mathrm{W} / \mathrm{C}=0.40 \mathrm{~S} / \mathrm{C}=4$} \\
\hline 4.2 .2 & 0.02 & 108.683 & 5.434 & 1553.25 & & & 0.02 & 103.48 & & 1034.77 & 69 \\
\hline 4.2. & 80 & 88.618 & & & & & 0.03 & 85.13 & & & \\
\hline 4.2 .4 & 4 & 807 & 7 & & & 4 & 4 & 2.30 & & & \\
\hline 4.2 .5 & 0.05 & 64.720 & 8. & .00 & & .5 & 0.05 & 62.84 & & 38 & .09 \\
\hline 4.2 .6 & 06 & 57.031 & 8.555 & 1087.75 & & 6 & 0.06 & 55.57 & 335 & 555.64 & .91 \\
\hline \multicolumn{6}{|c|}{$\mathrm{W} / \mathrm{C}=0.45 \mathrm{~S} / \mathrm{C}=3.5$} & \multicolumn{6}{|c|}{$\mathrm{W} / \mathrm{C}=0.40 \mathrm{~S} / \mathrm{C}=4$} \\
\hline 4.3 .2 & 0.02 & 120.630 & 5.361 & 938.231 & 268 & 5.3 .2 & 0.02 & 114.93 & 5.108 & 1024.55 & 5.39 \\
\hline 4.3 .3 & 0.03 & 98.603 & 6.574 & 766.912 & 219.118 & 5.3 .3 & 0.03 & 94.76 & 6.317 & 842.29 & 210.57 \\
\hline 4.3 .4 & 0.04 & 83.378 & 7.411 & 648.498 & 185.285 & 5.3 .4 & 0.04 & 80.61 & 7.166 & 716.55 & 179.14 \\
\hline 4.3 .5 & 0.05 & 72.226 & 8.025 & 561.760 & 160.503 & 5.3 .5 & 0.05 & 70.14 & 7.793 & 623.48 & 155.87 \\
\hline 4.3.6 & 0.06 & 63.705 & 8.494 & 495.487 & 141.568 & 5.3 .6 & 0.06 & 62.08 & 8.277 & 551.81 & 137.95 \\
\hline
\end{tabular}

\section{Materials}

Cement: It is a Portland cement per specification ASTM C 150 [14].

Sand: It is the locally available sand from Zliten area Libya. The sieve analysis was carried out and the results showed that it was within the permissible limits according to BS882: 1992 [15] as shown in Table 2.

Table 2: Sieve analysis of Sand

\begin{tabular}{|c|c|c|}
\hline Sieve size $(\mathrm{mm})$ & $\begin{array}{c}\text { Cumulative } \\
\text { passing (\%) }\end{array}$ & BS882:1992 \\
\hline 2.36 & 85.50 & $80-100$ \\
\hline 1.18 & 73.05 & $70-100$ \\
\hline $600 \mu \mathrm{m}$ & 63.00 & $55-100$ \\
\hline $300 \mu \mathrm{m}$ & 25.00 & $5-70$ \\
\hline $150 \mu \mathrm{m}$ & 0.40 & $0-15$ \\
\hline
\end{tabular}

Polystyrene: It is a lightweight material that is easy to carry and transport with a white color in the form of small beads with a density ranging between $(10-27) \mathrm{kg} / \mathrm{m}^{3}$. The density of polystyrene used was $22.4 \mathrm{~kg} / \mathrm{m}^{3}$ and Fig. 1 shows a sample from polystyrene beads used.

Mixing and curing water: Drinking water was used free of organic matter, salts and impurities.

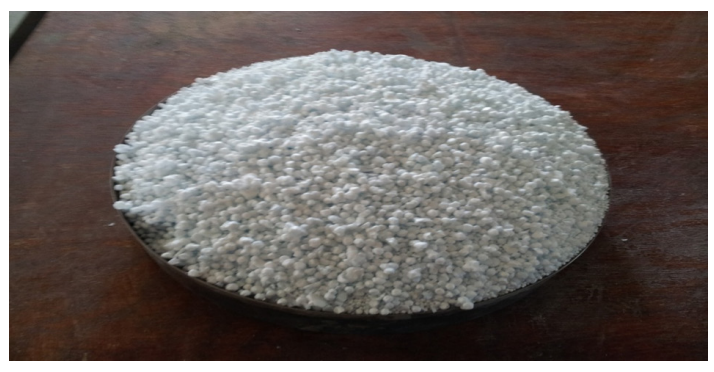

Figure 1: Polystyrene beads

\section{Mixing, Casting and testing of concrete specimens}

Sand and cement were placed in the mixing bowl, and they were mixed manually in dry condition until the mix became homogeneous. Then the polystyrene was added with continuous mixing, and with gradual addition of water. The mixing was done manually to ensure that the polystyrene beads do not fly out. For compressive strength and dry density tests, $(100 \times 100 \times 100) \mathrm{mm}$ cubes were used. After completion of concrete pouring in the cubes as shown in Fig 2, the concrete surfaces were leveled and left for 24 hours to harden, then the molds were dismantled and the concrete cubes were curried in water tank for 28 days, as shown in Figs 3 . Compressive strength test has been done as per B.S.1881, Part 116, 1989 [16] as shown in Figs. (4). Dry density test has been done as per ASTM C 642 [17].

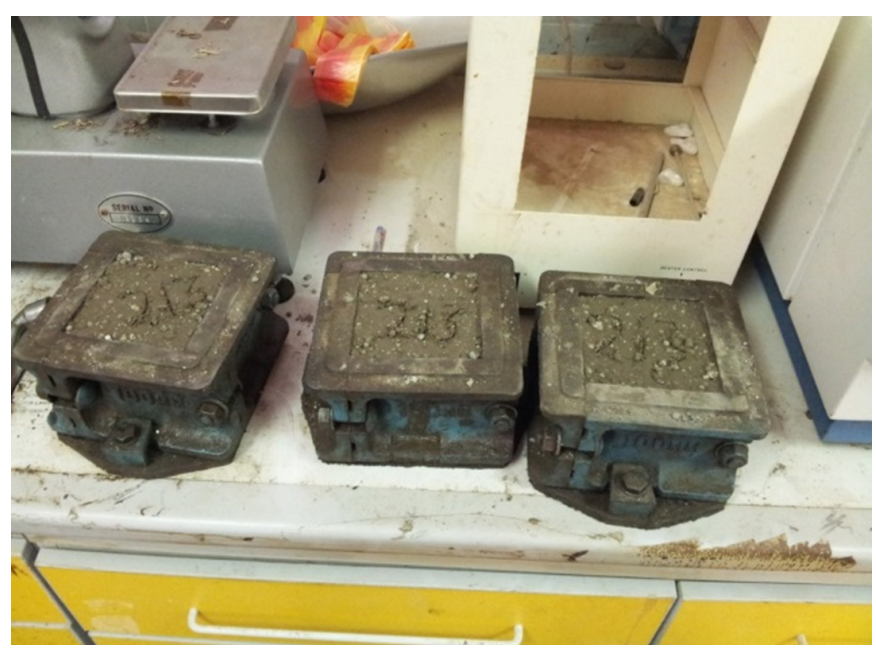

Figure 2: Casting of concrete 


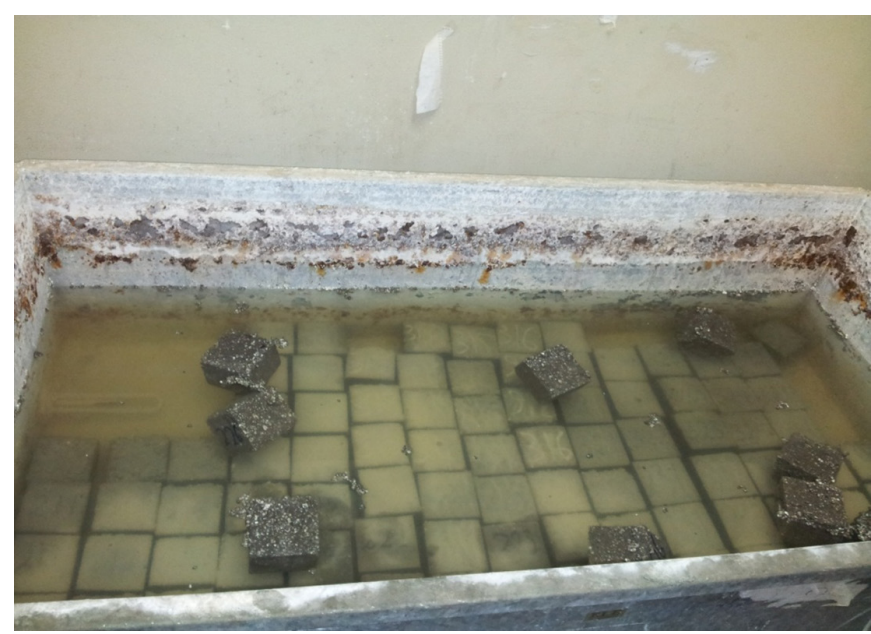

Figure 3: Curing of concrete

\section{RESULTS AND DISCUSSION}

Test results of both compressive strength and dry density for specimens cast with different ratios and after the curing period 28 days were determined and are presented in Table 3 as well as in Figures 5 to 8.

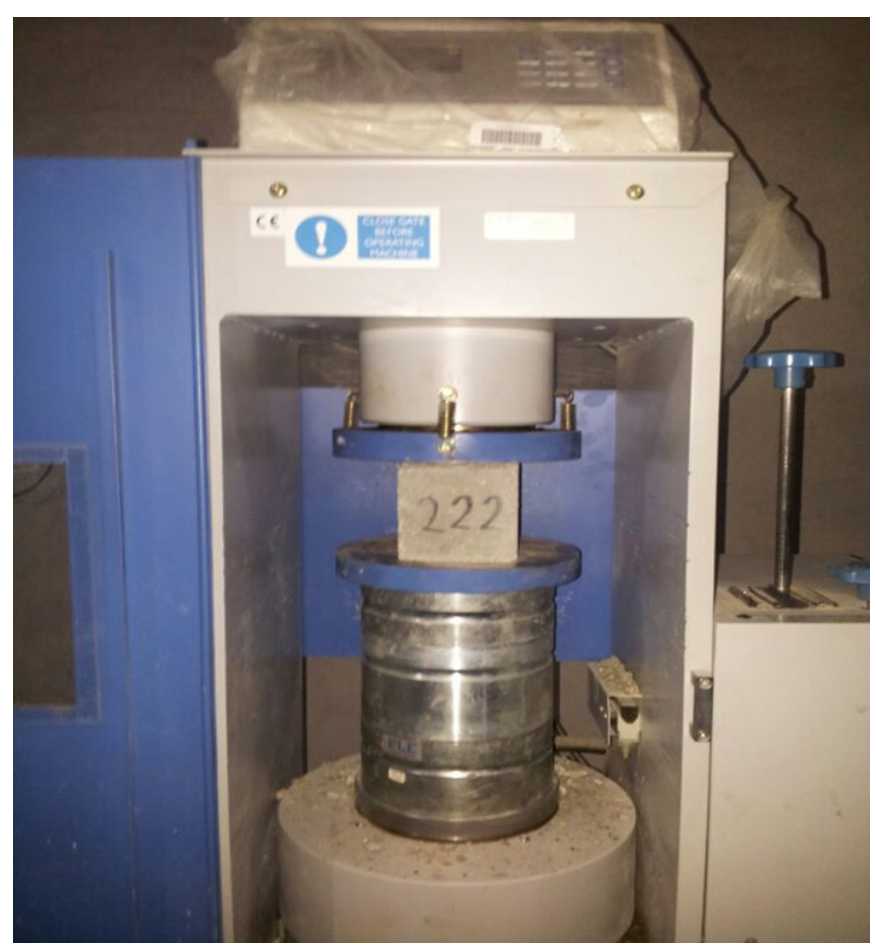

Figure 4: Compression test device during the test

Table 3: Tests results polystyrene lightweight concrete

\begin{tabular}{|c|c|c|c|c|c|c|c|c|c|c|c|}
\hline $\begin{array}{l}\text { Mix } \\
\text { No. }\end{array}$ & P/C & $\begin{array}{c}\text { Com- } \\
\text { pressive } \\
\text { Strength } \\
\text { Mpa }\end{array}$ & $\begin{array}{c}\text { Reduction } \\
\text { in Com- } \\
\text { pressive } \\
\text { Strength }\end{array}$ & $\begin{array}{c}\text { Dry } \\
\text { Density } \\
\mathrm{Kg} / \mathrm{m}^{3}\end{array}$ & $\begin{array}{c}\text { Reduc- } \\
\text { tion in } \\
\text { Density } \\
(\%)\end{array}$ & $\begin{array}{l}\text { Mix } \\
\text { No. }\end{array}$ & $\mathrm{P} / \mathrm{C}$ & $\begin{array}{c}\text { Com- } \\
\text { pressive } \\
\text { Strength } \\
\text { Mpa }\end{array}$ & $\begin{array}{l}\text { Reduction } \\
\text { in Com- } \\
\text { pressive } \\
\text { Strength }\end{array}$ & $\begin{array}{c}\text { Dry } \\
\text { Density } \\
\mathrm{Kg} / \mathrm{m}^{3}\end{array}$ & $\begin{array}{l}\text { Reduc- } \\
\text { tion in } \\
\text { Density } \\
(\%)\end{array}$ \\
\hline \multicolumn{6}{|c|}{$\mathrm{W} / \mathrm{C}=0.35 \mathrm{~S} / \mathrm{C}=2.5$} & \multicolumn{6}{|c|}{$W / C=0.35 \mathrm{~S} / \mathrm{C}=3$} \\
\hline 2.1 .2 & 0.02 & 4.35 & & 1287.8 & 0 & 3.1 .2 & 0.02 & 3.13 & 0 & 1450.3 & 0 \\
\hline 2.1 .3 & 0.03 & 2.08 & 52.18 & 1081.0 & 16.06 & 3.1 .3 & 0.03 & 1.74 & 44.41 & 1293.0 & 10.84 \\
\hline 2.1 .4 & 0.04 & 1.35 & 68.97 & 900.5 & 30.07 & 3.1 .4 & 0.04 & 1.21 & 61.34 & 1073.0 & 26.02 \\
\hline 2.1 .5 & 0.05 & 0.82 & 81.15 & 853.3 & 33.74 & 3.1 .5 & 0.05 & 0.63 & 79.87 & 966.0 & 33.39 \\
\hline 2.1 .6 & 0.06 & 0.59 & 86.44 & 777.0 & 39.94 & 3.1 .6 & 0.06 & 0.55 & 82.43 & 934.8 & 35.54 \\
\hline \multicolumn{6}{|c|}{$\mathrm{W} / \mathrm{C}=0.40 \mathrm{~S} / \mathrm{C}=2.5$} & \multicolumn{6}{|c|}{$\mathrm{W} / \mathrm{C}=0.40 \mathrm{~S} / \mathrm{C}=3$} \\
\hline 2.2 .2 & 0.02 & 4.95 & 0 & 1472.0 & 0 & 3.2 .2 & 0.02 & 3.67 & 0 & 1414.5 & 0 \\
\hline 2.2 .3 & 0.03 & 2.62 & 47.07 & 1189.0 & 19.23 & 3.2 .3 & 0.03 & 1.51 & 58.86 & 1229.0 & 13.11 \\
\hline 2.2 .4 & 0.04 & 1.78 & 64.04 & 1014.3 & 31.09 & 3.2 .4 & 0.04 & 0.88 & 76.02 & 1124.8 & 20.48 \\
\hline 2.2 .5 & 0.05 & 0.93 & 81.21 & 940.0 & 36.14 & 3.2 .5 & 0.05 & 0.67 & 81.74 & 935.8 & 33.84 \\
\hline 2.2 .6 & 0.06 & 0.58 & 88.08 & 792.3 & 46.18 & 3.2 .6 & 0.06 & 0.38 & 89.16 & 847.5 & 40.08 \\
\hline \multicolumn{6}{|c|}{$\mathrm{W} / \mathrm{C}=0.45 \mathrm{~S} / \mathrm{C}=2.5$} & \multicolumn{6}{|c|}{$\mathrm{W} / \mathrm{C}=0.45 \mathrm{~S} / \mathrm{C}=3$} \\
\hline 2.3 .2 & 0.02 & 5.93 & 0 & 1462.3 & 0 & 3.2 .2 & 0.02 & 2.35 & 0 & 1390.5 & 0 \\
\hline 2.3 .3 & 0.03 & 2.94 & 50.42 & 1212.3 & 17.10 & 3.2 .3 & 0.03 & 2.32 & 1.28 & 1212.0 & 12.84 \\
\hline 2.3 .4 & 0.04 & 2.51 & 57.67 & 1117.8 & 23.54 & 3.2 .4 & 0.04 & 1.15 & 51.06 & 1102.8 & 20.71 \\
\hline 2.3 .5 & 0.05 & 1.39 & 76.56 & 912.5 & 37.60 & 3.2 .5 & 0.05 & 0.53 & 77.45 & 905.8 & 34.86 \\
\hline 2.3 .6 & 0.06 & 0.65 & 89.04 & 913.5 & 37.52 & 3.2 .6 & 0.06 & 0.38 & 83.83 & 827.5 & 40.49 \\
\hline
\end{tabular}




\begin{tabular}{|c|c|c|c|c|c|c|c|c|c|c|c|}
\hline $\begin{array}{l}\text { Mix } \\
\text { No. }\end{array}$ & $\mathrm{P} / \mathrm{C}$ & $\begin{array}{c}\text { Com- } \\
\text { pressive } \\
\text { Strength } \\
\text { Mpa }\end{array}$ & $\begin{array}{l}\text { Reduction } \\
\text { in Com- } \\
\text { pressive } \\
\text { Strength }\end{array}$ & $\begin{array}{c}\text { Dry } \\
\text { Density } \\
\mathrm{Kg} / \mathrm{m}^{3}\end{array}$ & $\begin{array}{c}\text { Reduc- } \\
\text { tion in } \\
\text { Density } \\
(\%)\end{array}$ & $\begin{array}{l}\text { Mix } \\
\text { No. }\end{array}$ & $\mathrm{P} / \mathrm{C}$ & \begin{tabular}{|c|} 
Com- \\
pressive \\
Strength \\
Mpa
\end{tabular} & $\begin{array}{l}\text { Reduction } \\
\text { in Com- } \\
\text { pressive } \\
\text { Strength }\end{array}$ & $\begin{array}{c}\text { Dry } \\
\text { Density } \\
\mathrm{Kg} / \mathrm{m}^{3}\end{array}$ & $\begin{array}{c}\text { Reduc- } \\
\text { tion in } \\
\text { Density } \\
(\%)\end{array}$ \\
\hline \multicolumn{6}{|c|}{$\mathrm{W} / \mathrm{C}=0.35 \mathrm{~S} / \mathrm{C}=3.5$} & \multicolumn{6}{|c|}{$\mathrm{W} / \mathrm{C}=0.35 \mathrm{~S} / \mathrm{C}=4$} \\
\hline 4.1 .2 & 0.02 & 3.35 & 0 & 1538.0 & 0 & 5.1 .2 & 0.02 & 1.30 & 0 & 1461.5 & 0 \\
\hline 4.1 .3 & 0.03 & 153 & 54.32 & 1372.5 & 10.76 & 5.1 .3 & 0.03 & & 32.31 & 1262.3 & 13.63 \\
\hline 4.1 .4 & 0.04 & & 70.75 & 117 & 34 & 5.1 .4 & 0.04 & & & & \\
\hline 4.1 .5 & 0.05 & & & 1099.0 & 4 & 5.1 .5 & 0.05 & & & 0.5 & 07 \\
\hline 4.1 .6 & 0.06 & 0.42 & 87.46 & 980.8 & & 5.1 .6 & 0.06 & 0.33 & 74.62 & & \\
\hline \multicolumn{6}{|c|}{$\mathrm{W} / \mathrm{C}=0.40 \mathrm{~S} / \mathrm{C}=3.5$} & \multicolumn{6}{|c|}{$\mathrm{W} / \mathrm{C}=0.40 \mathrm{~S} / \mathrm{C}=4$} \\
\hline 2.2 & 0.02 & 2.67 & 0 & 1553.3 & 0 & 5.2 .2 & .02 & 1.54 & 0 & 1419.5 & 0 \\
\hline 4.2 .3 & 0.03 & & .2 & 1248.8 & 19.60 & 5.2 .3 & 0.03 & 1 & & & 7.62 \\
\hline 4.2 .4 & 0.04 & & 5 & 1178.0 & 16 & 5.2 .4 & 0.04 & 0. & & .0 & 18.21 \\
\hline 4.2 .5 & 0.05 & & & 10 & 5 & 5.2 .5 & 0.05 & 0. & & & 23.02 \\
\hline 4.2 .6 & 0.06 & 0.49 & 81.65 & 1087.8 & 29.97 & 5.2 .6 & 0.06 & 0.44 & 71.43 & 1038.0 & 26.88 \\
\hline \multicolumn{6}{|c|}{$\mathrm{W} / \mathrm{C}=0.45 \mathrm{~S} / \mathrm{C}=3.5$} & \multicolumn{6}{|c|}{$\mathrm{W} / \mathrm{C}=0.45 \mathrm{~S} / \mathrm{C}=4$} \\
\hline 4.3 .2 & 0.02 & 2.49 & 0 & 1519.0 & 0 & 5.3 .2 & 0.02 & 1.85 & 0 & 1509.5 & 0 \\
\hline 4.3 .3 & 0.03 & 1.93 & 22.49 & 1296.3 & 14.66 & 5.3 .3 & 0.03 & 1.26 & 31.89 & 1323.0 & 12.36 \\
\hline 4.3 .4 & 0.04 & 1.14 & 54.22 & 1149.3 & 24.34 & 5.3 .4 & 0.04 & 0.97 & 47.57 & 1220.0 & 19.18 \\
\hline 4.3 .5 & 0.05 & 0.84 & 66.27 & 1076.3 & 29.14 & 5.3 .5 & 0.05 & 0.53 & 71.35 & 1082.5 & 28.29 \\
\hline 4.3 .6 & 0.06 & 0.69 & 72.29 & 1090.5 & 28.21 & 5.3 .6 & 0.06 & 0.51 & 72.43 & 972.3 & 35.59 \\
\hline
\end{tabular}

\section{Compressive strength}

Figs 5 and 6 represent the relationship between compressive strength and polystyrene content for different $(\mathrm{S} / \mathrm{C})$ ratio, and $(\mathrm{W} / \mathrm{C})$ ratio. The compressive strength decreases as the $(\mathrm{P} / \mathrm{C})$ ratio increases. For all mixes the maximum decrease occurred when the $(\mathrm{P} / \mathrm{C})$ ratio was increased from 0.02 to 0.03 , with average reduction of $37.55 \%$. While, when the $(\mathrm{P} / \mathrm{C})$ ratio was increased from 0.03 to 0.04 , from 0.04 to 0.05 and from 0.05 to 0.06 , the average reduction was $21.21 \%, 14.59 \%$ and $8.19 \%$, respectively. Also it was found that compressive strength is more sensitive to the polystyrene content than dry density as the reduction in compressive strength reached about $82 \%$ in comparison with $36 \%$ for dry density when the $(P / C)$ ratio was increased from 0.02 to 0.06 . This may be attributed to the weak bond between polystyrene beads and cement paste, and the weakness of the bead themselves.

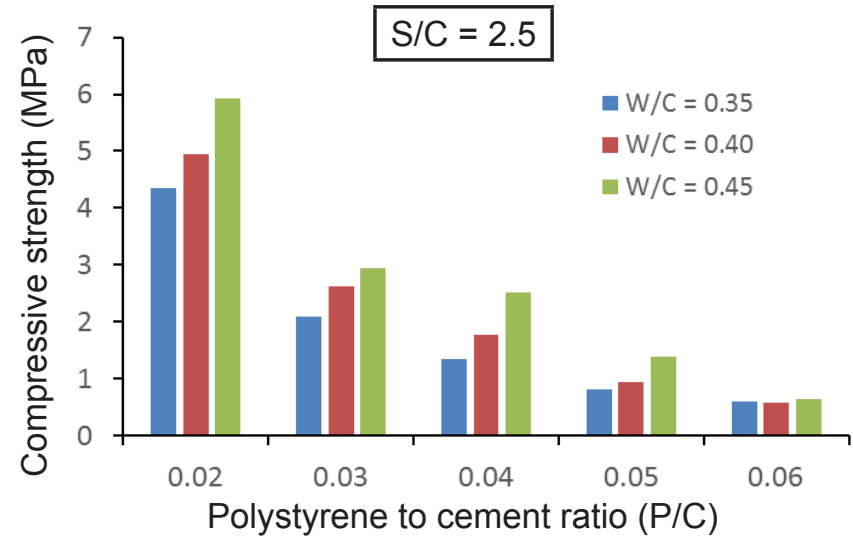

(a)
Compressive strength decreases as the $(\mathrm{S} / \mathrm{C})$ ratio increases for specified $(\mathrm{W} / \mathrm{C})$ and $(\mathrm{P} / \mathrm{C})$ ratios in the mixture. As an example, for (W/C) ratio equal to 0.35 , the increase of $(\mathrm{S} / \mathrm{C})$ ratio from 2.5 to 3 resulted in reductions of $28.05 \%, 16.34 \%, 10.37 \%, 23.17 \%$, and $6.78 \%$ for $(P / C)$ ratios $0.02,0.03,0.04,0.05$ and 0.06 , respectively, and the increase of $(\mathrm{S} / \mathrm{A})$ ratio from 3 to 3.5 caused reductions of $-7.03 \%$ (increasing), 12.07\%, 19.00\%, 17.46\%, and $23.63 \%$ for $(\mathrm{P} / \mathrm{C})$ ratios $0.02,0.03$ and 0.040 .05 and 0.06 , respectively, while the change of $(\mathrm{S} / \mathrm{C})$ ratio from 3.5 to 4 , resulted in reductions of $61.19 \%, 42.48 \%$, $37.76 \%,-3.80 \%$, and $21.43 \%$ for $(P / C)$ ratios $0.02,0.03$, 0.040 .05 and 0.06 , respectively.

A considerable number of mixes showed an increase in the compressive strength when the (W/C) ratio was increased in addition the obtained maximum measured value of was at $(\mathrm{W} / \mathrm{C})$ ratio equal to 0.45 . This may be attributed to the good compaction achieved in this mixture.

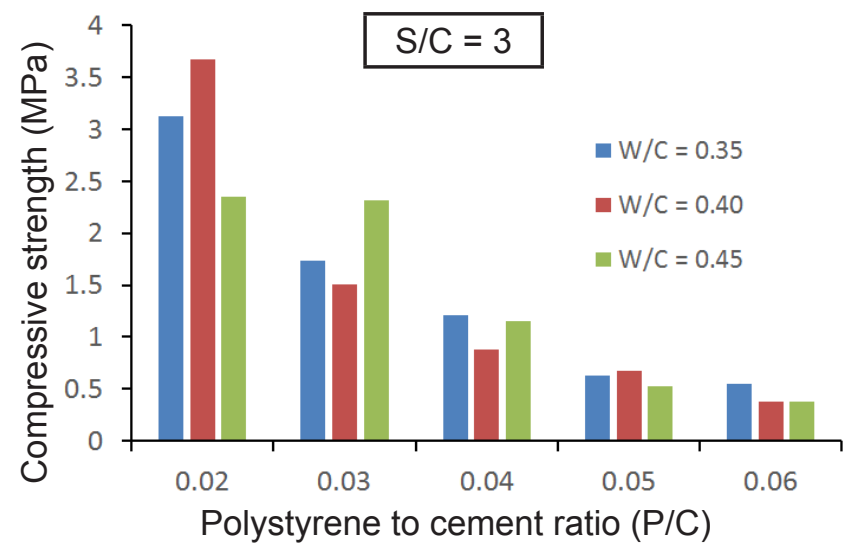

(b) 


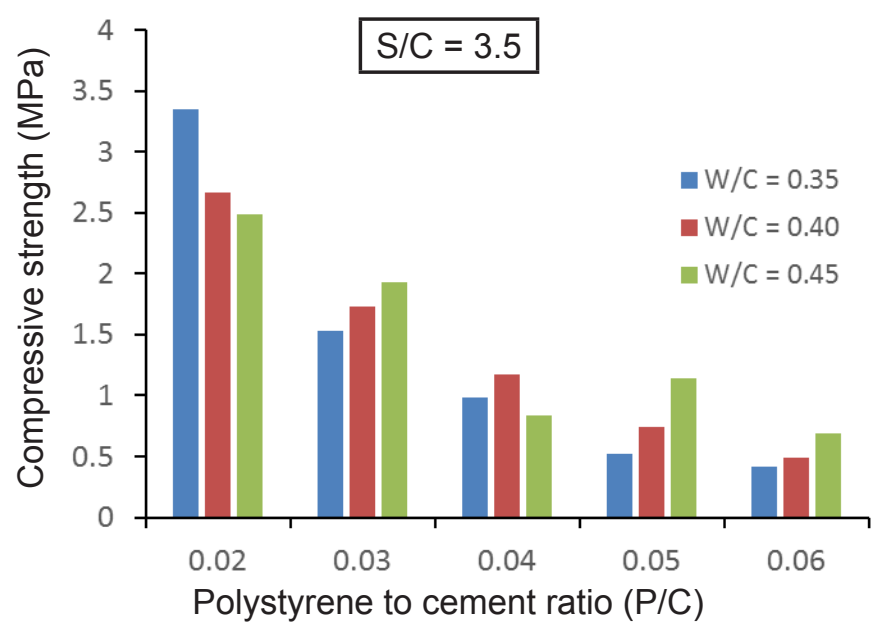

(c)

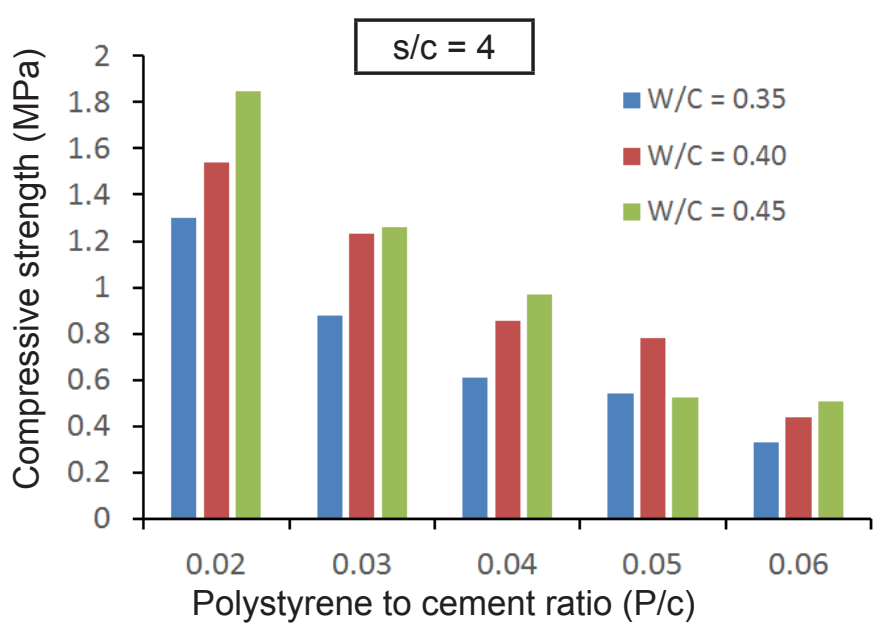

(d)

Figure 5: Variation of compressive strength with $(P / C),(S / C)$, and $(W / C)$

Fig. 6 shows the correlation between compressive strength and polystyrene to cement ratio for mixtures with different $(\mathrm{S} / \mathrm{C})$ and $(\mathrm{W} / \mathrm{C})$ ratios. Considerably high relationships were obtained between compressive strength and $(P / C)$

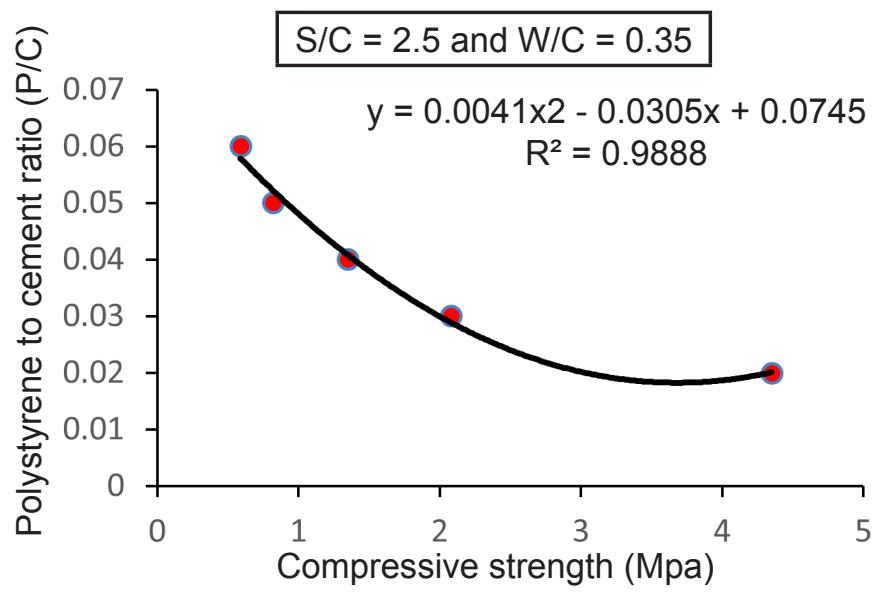

(a)

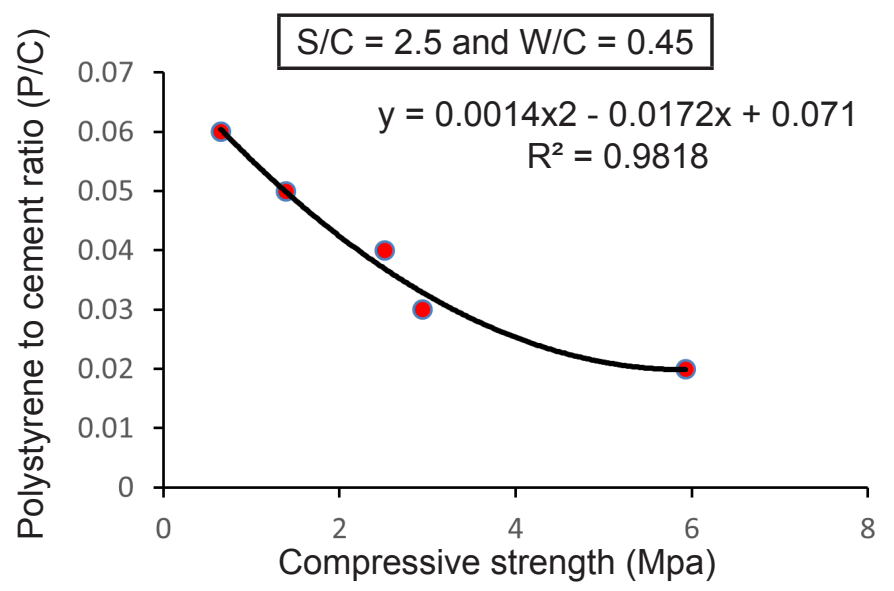

(c) ratio as the regression analysis provided an average correlation coefficient (R2) of 0.9751 , so, they can be of use in determining mix proportions of polystyrene lightweight concrete based on the required compressive strength.

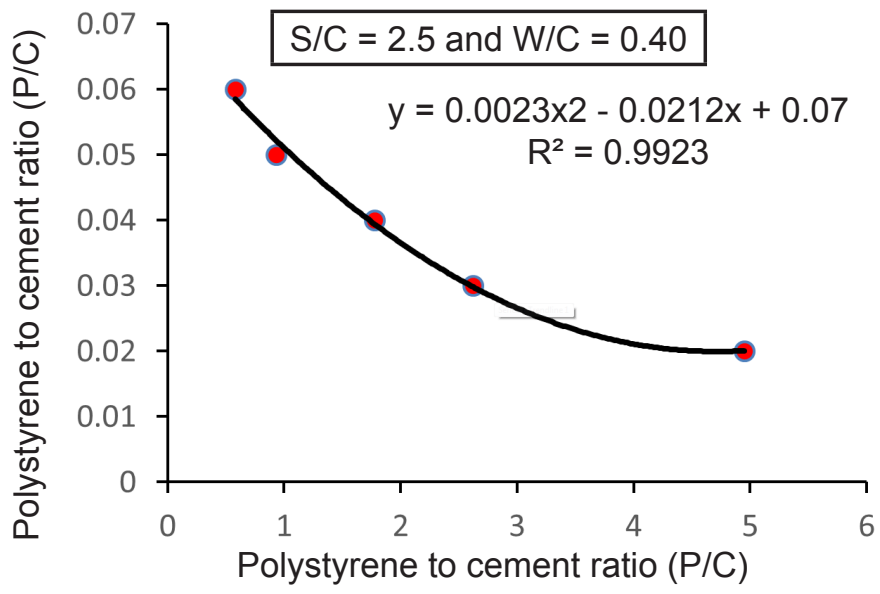

(b)

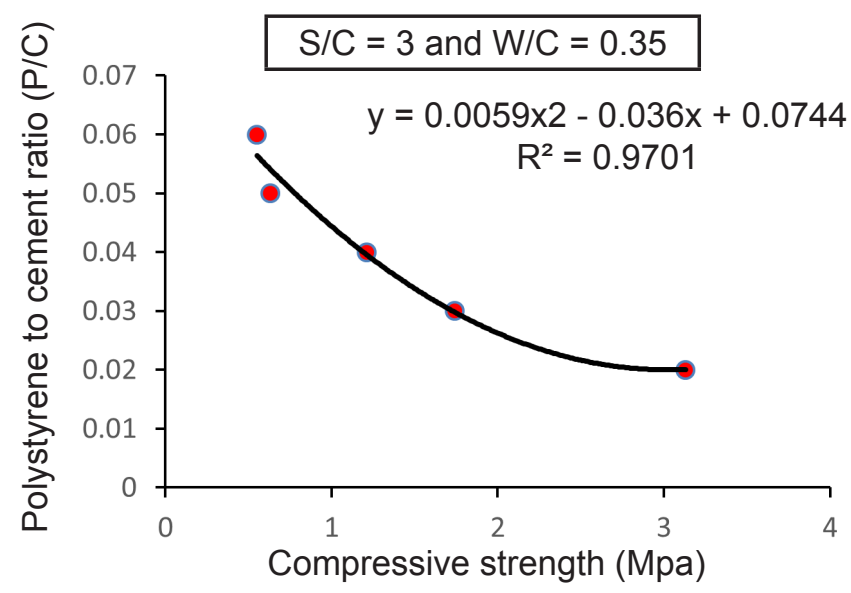

(d) 


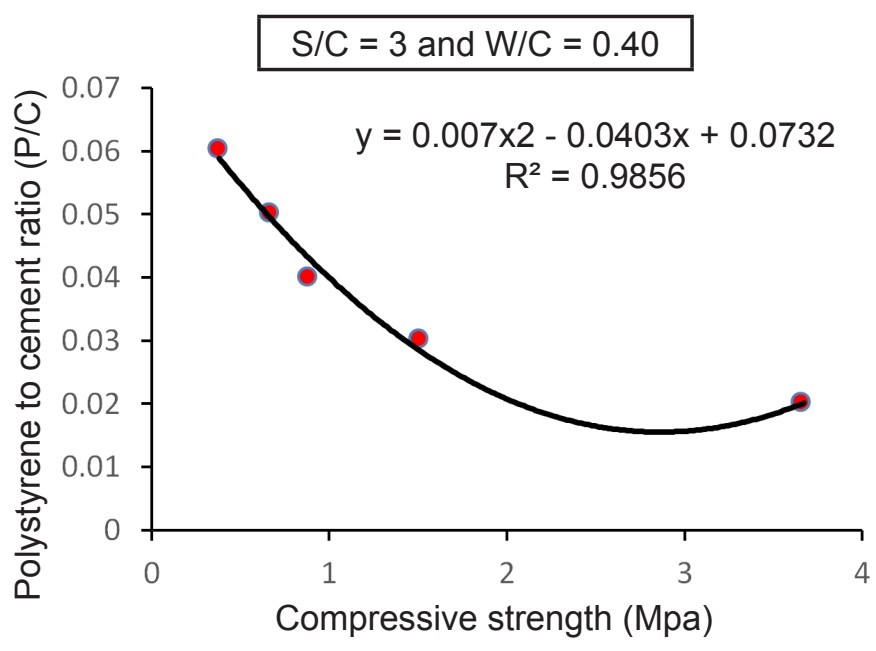

(e)

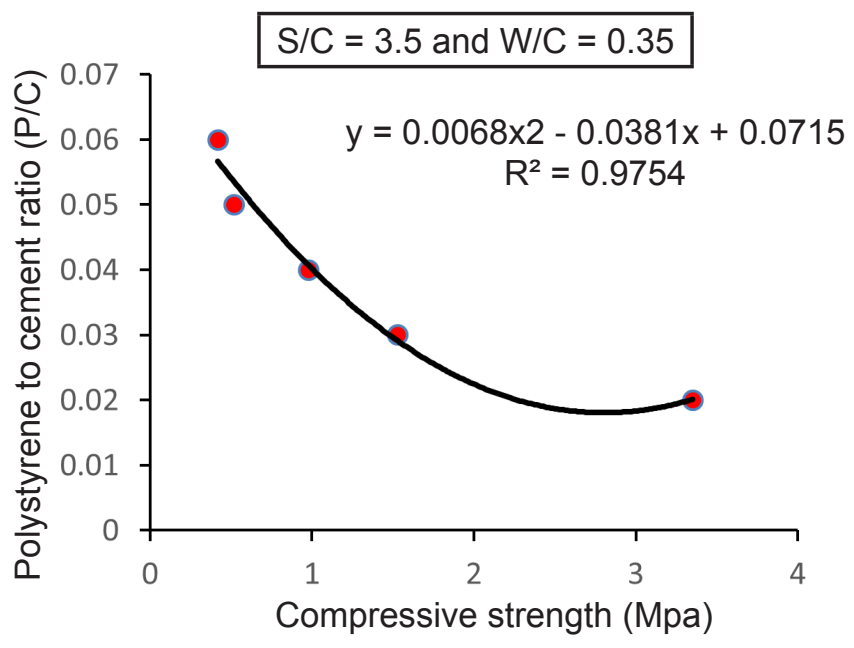

(g)

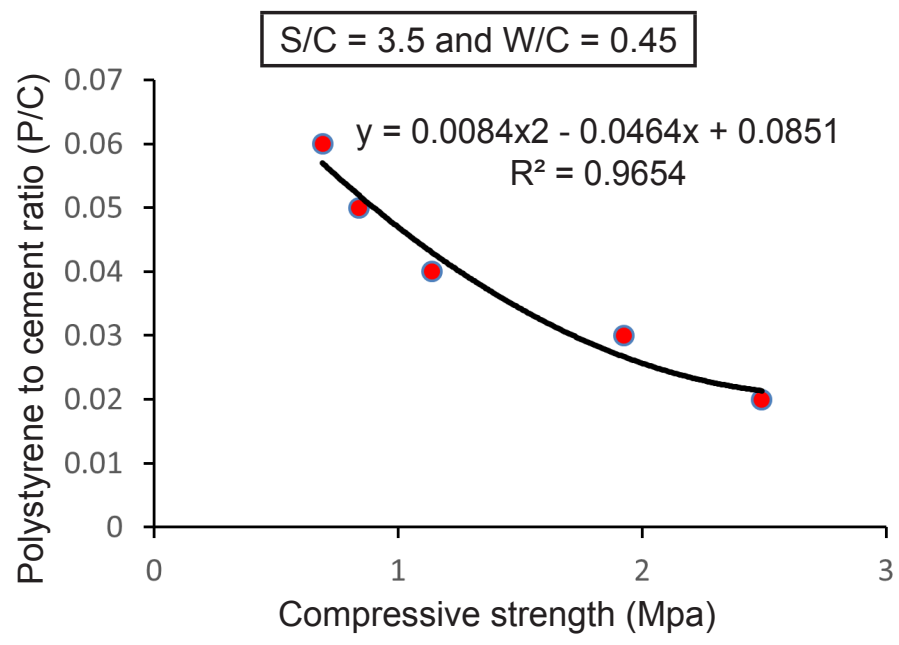

(i)

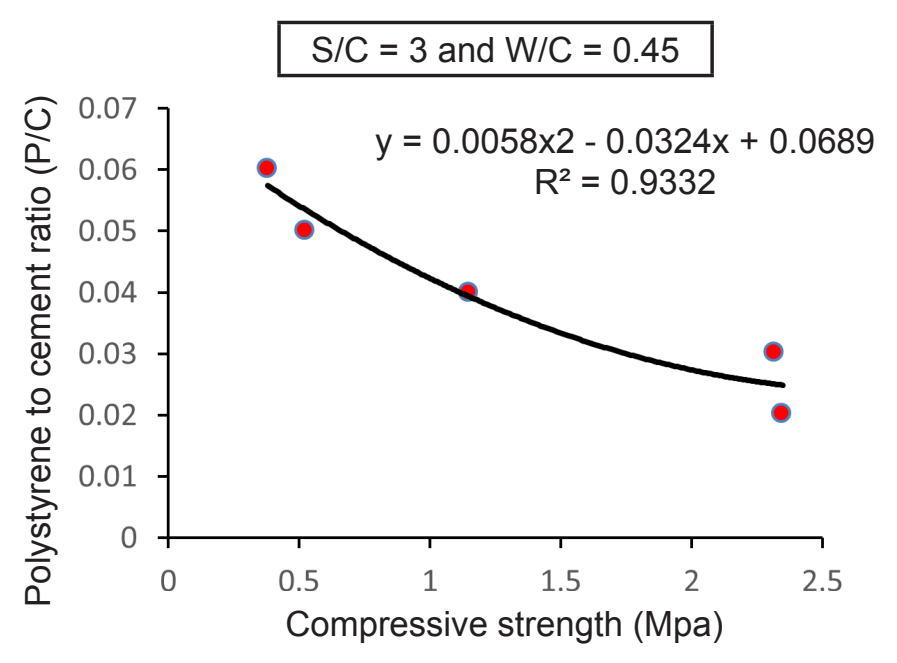

(f)

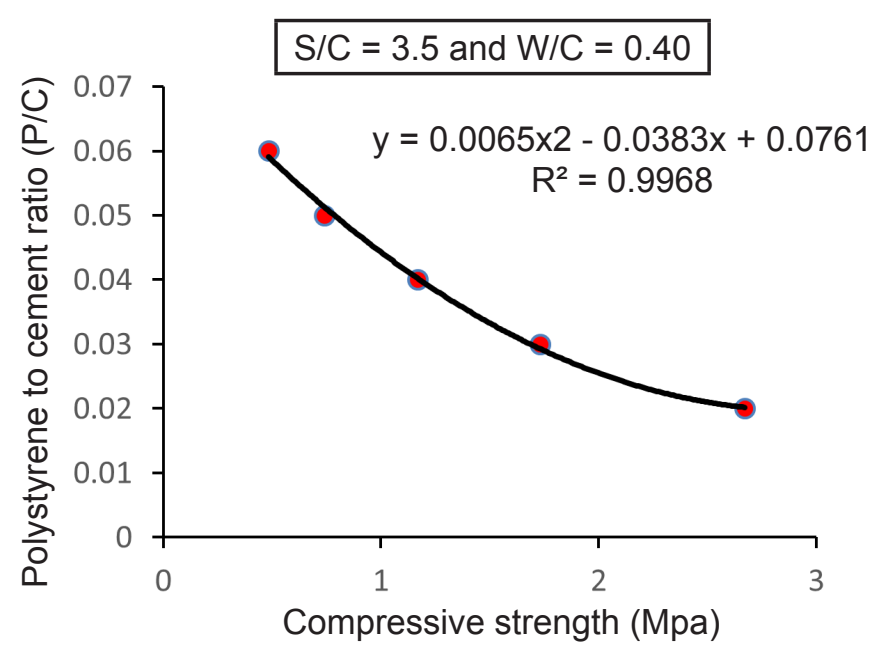

(h)

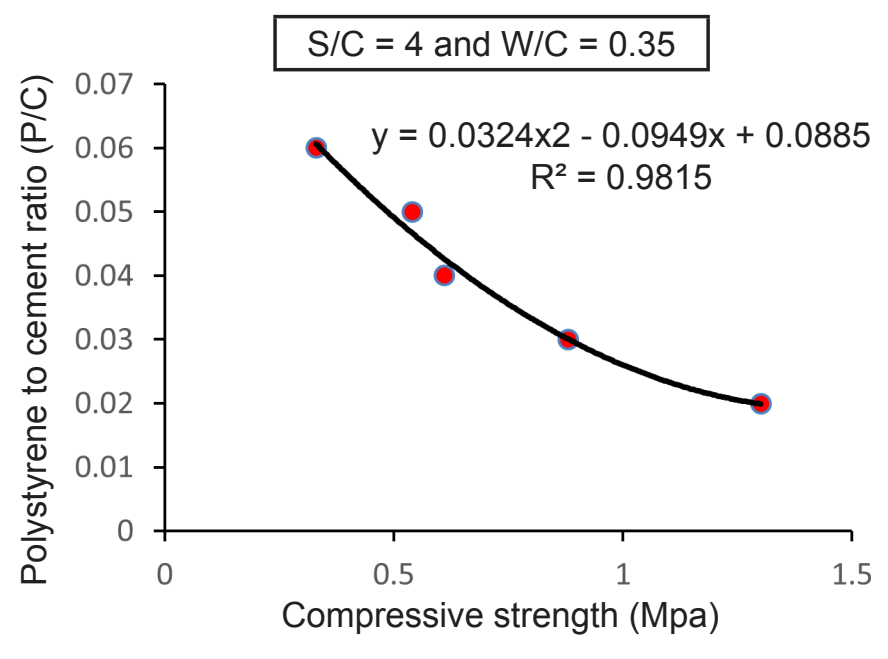

(j) 


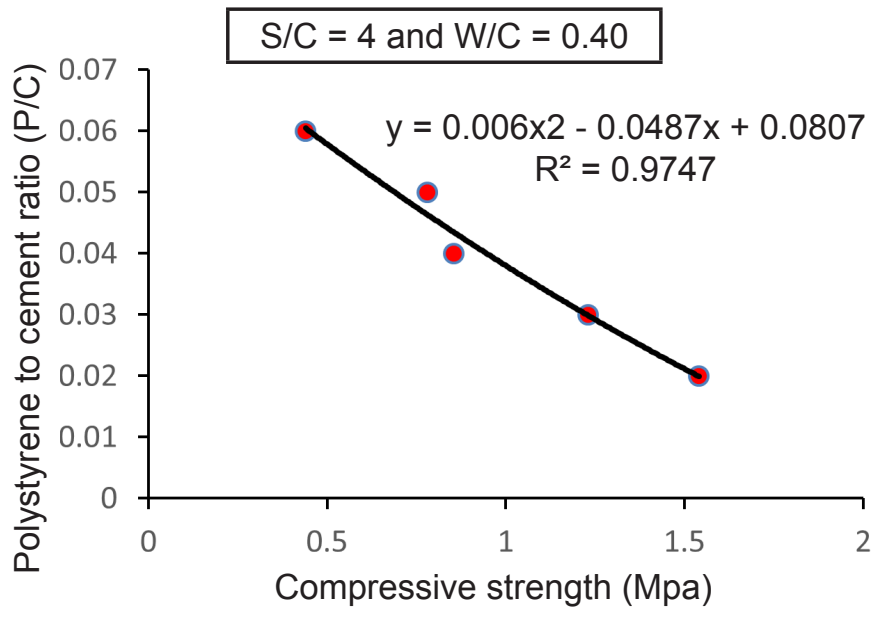

(k)

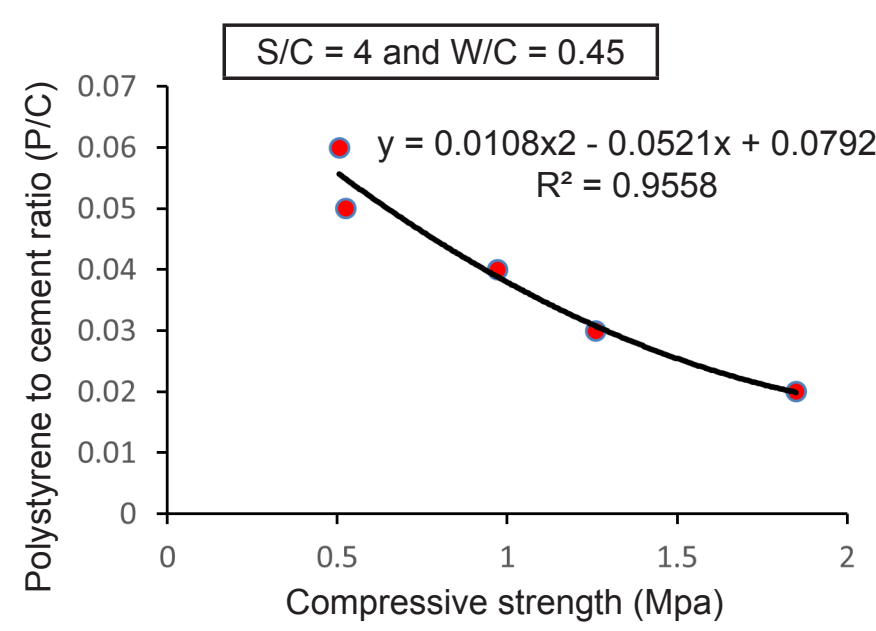

(l)

Figure 6: The relationships between compressive strength and $(P / C)$ ratios

\section{Dry density}

Fig 7 and 8 show the relationship between dry density and polystyrene content for different $(\mathrm{S} / \mathrm{C})$ and $(\mathrm{W} / \mathrm{c})$ ratios. The results presented in diagrams in Figure 7 and Table 3 show the expected increasing and decreasing trends for dry density with the increase of $(\mathrm{S} / \mathrm{C})$ ratio and

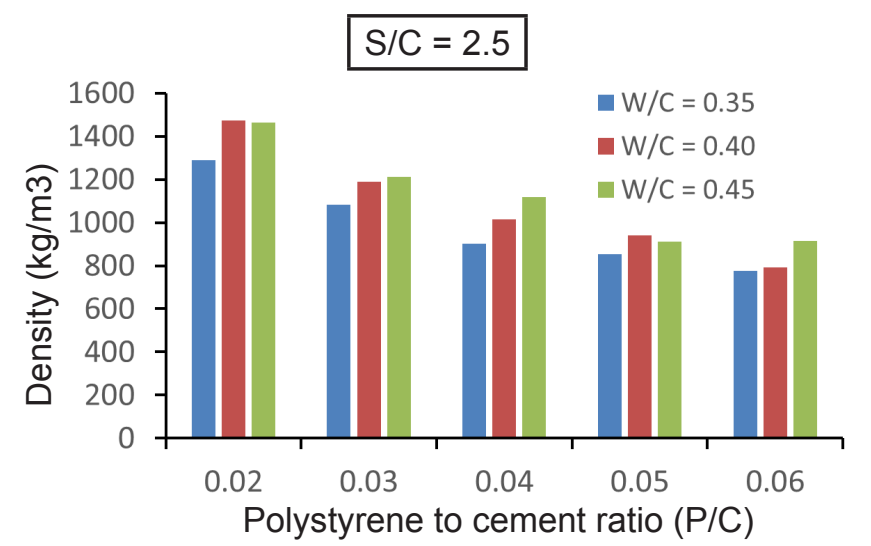

(a)

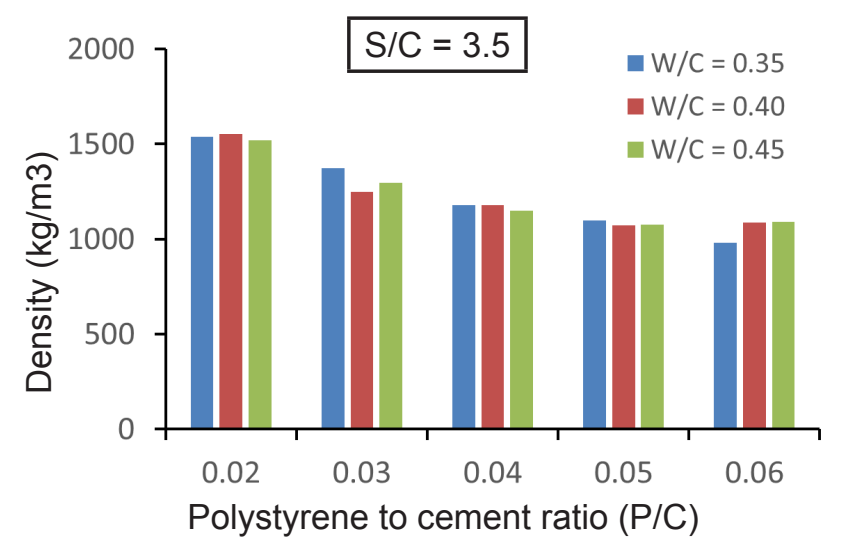

(c) the decrease of $(P / C)$ ratio in the mixture, respectively. The density of the concrete depends upon mix proportions, compaction and curing conditions. For the tested mixtures, the results show that dry density values are ranging from $777.0 \mathrm{~kg} / \mathrm{m}^{3}$ to $1553.3 \mathrm{~kg} / \mathrm{m}^{3}$ and the reduction values in the dry density due the variation of mix proportions are ranging from $7.62 \%$ to $46.18 \%$.

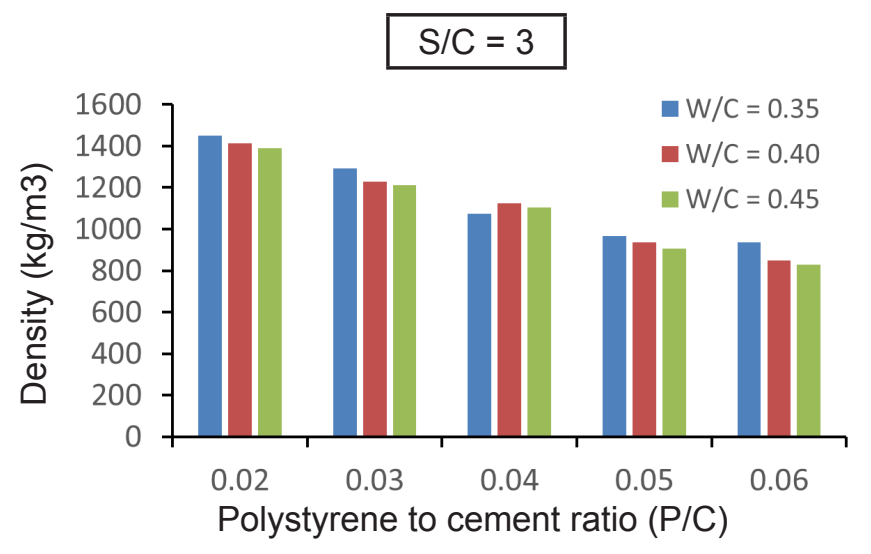

(b)

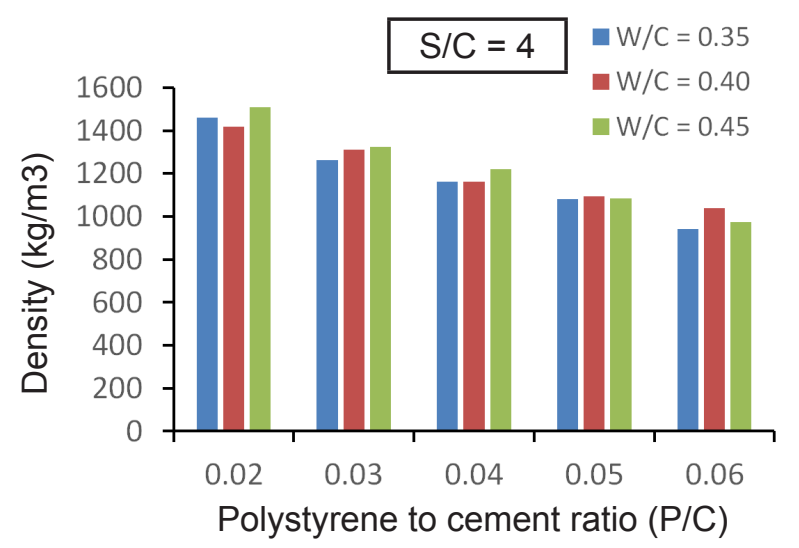

(d)

Figure 7: Variation of density with $(P / C),(W / C)$, and $(S / C)$ 
Fig. 8 shows the correlation between dry density and polystyrene to cement ratio for mixtures with different sand and water contents. A Considerably high relationships were obtained between dry densities and $(\mathrm{P} / \mathrm{C})$ ratio for poly-

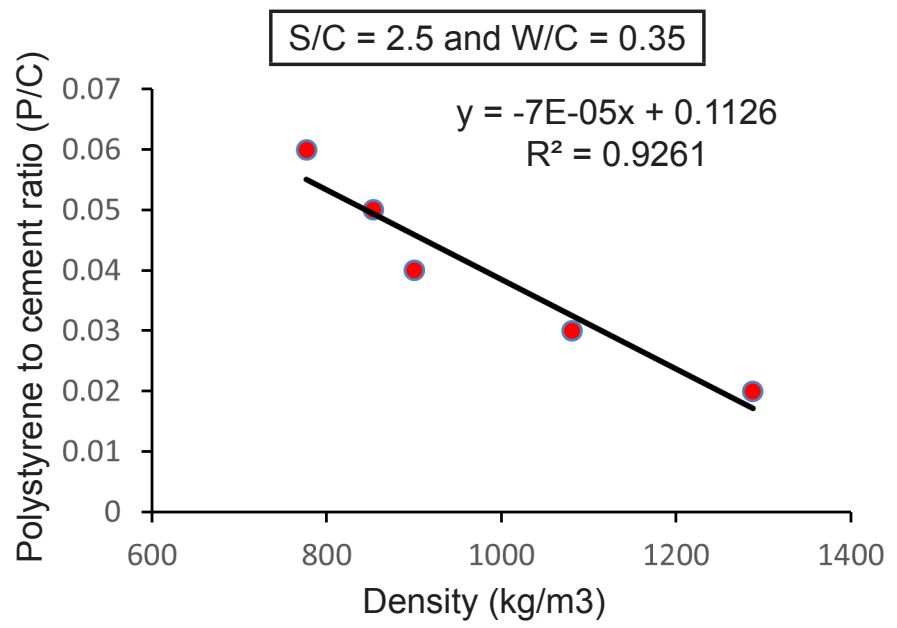

(a)

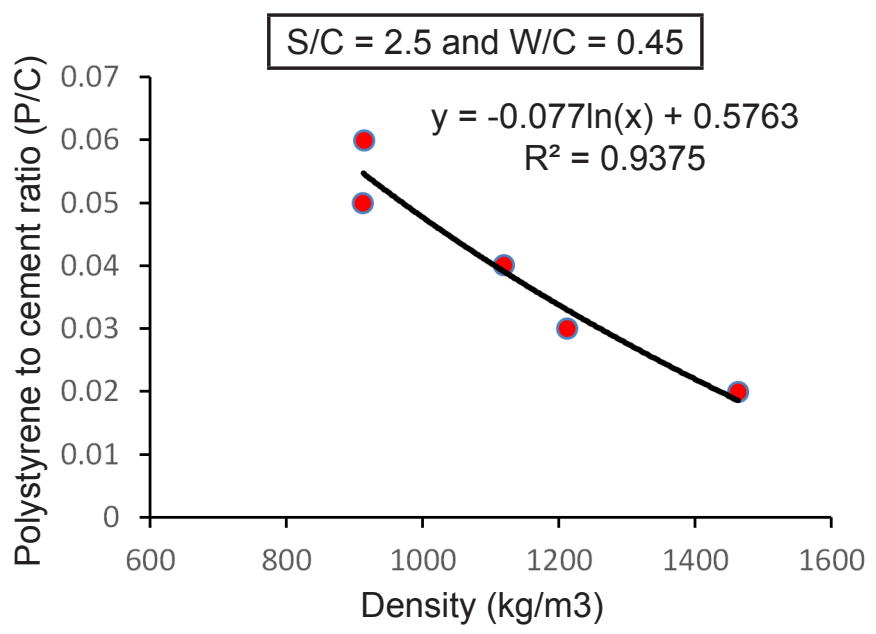

(c)

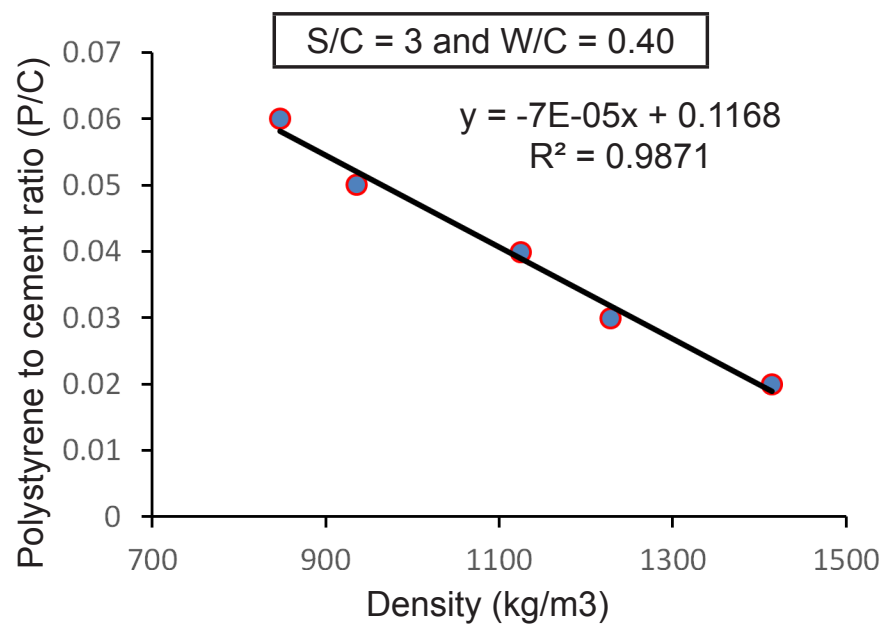

(e) styrene lightweight concrete as the regression analysis provided an average correlation coefficient $\left(R^{2}\right)$ of 0.9401 . So, they can be used to determine the proportion of the mixture components based on the required dry density.

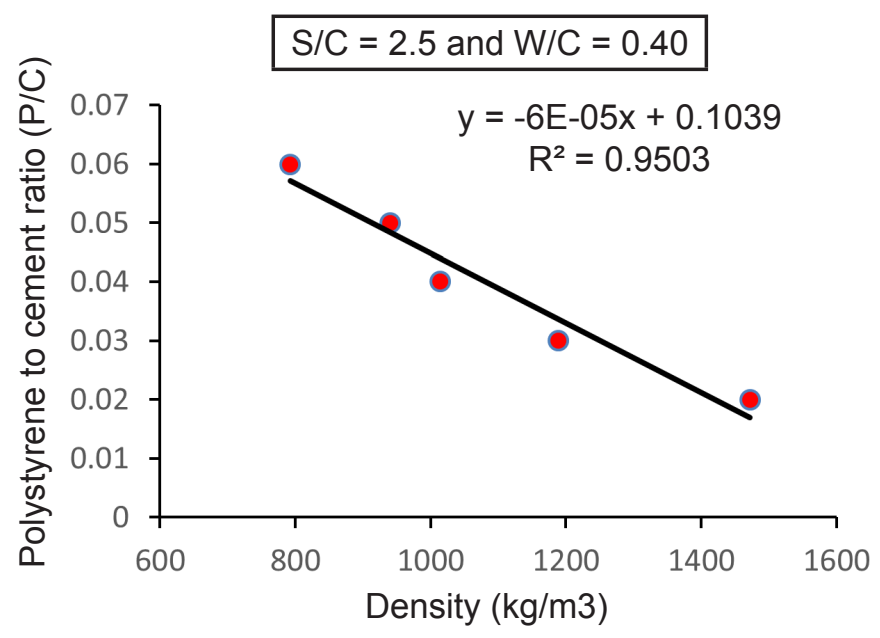

(b)

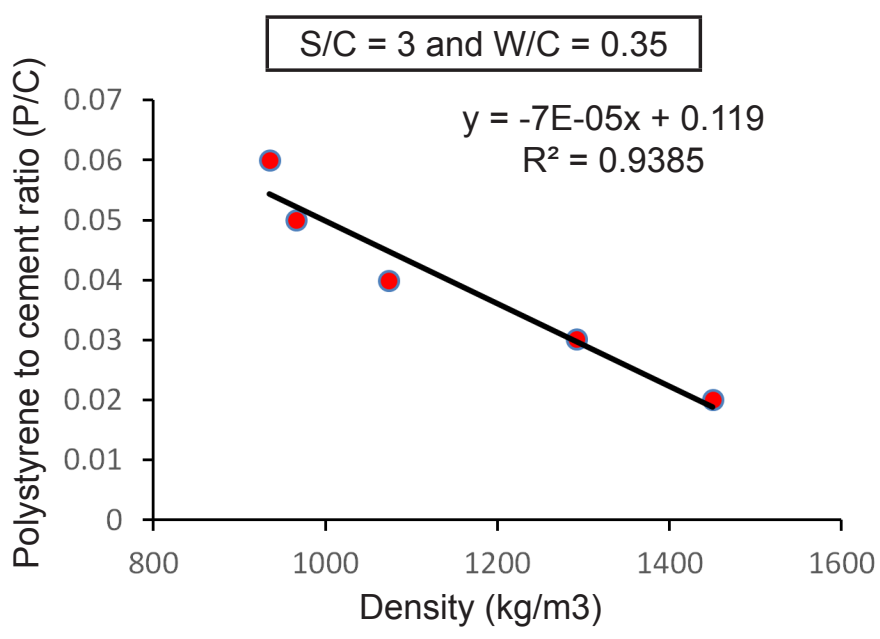

(d)

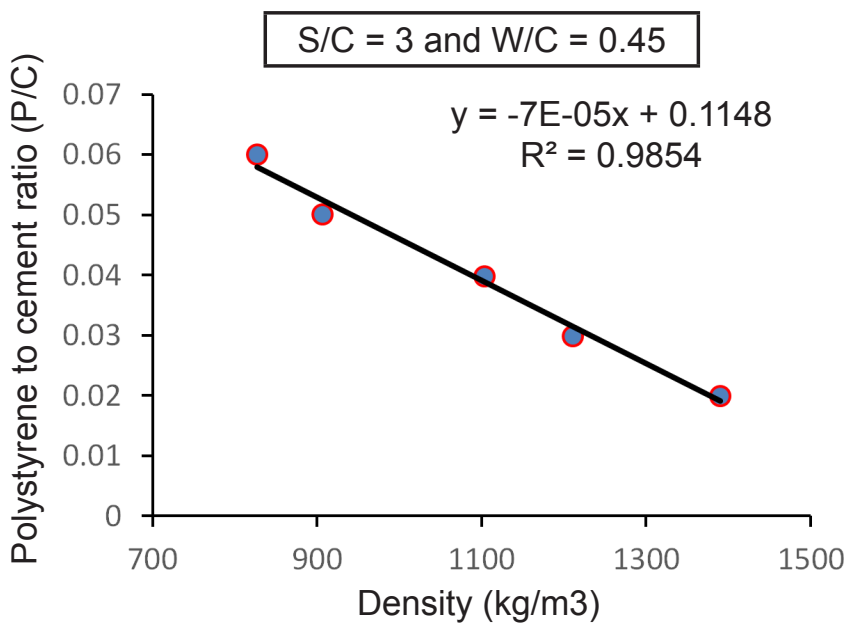

(f) 


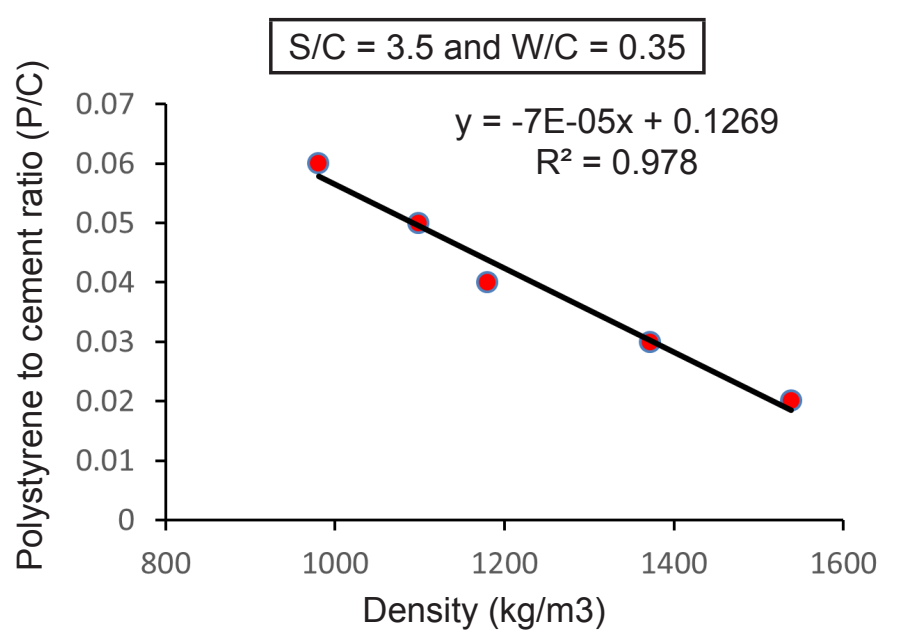

(g)

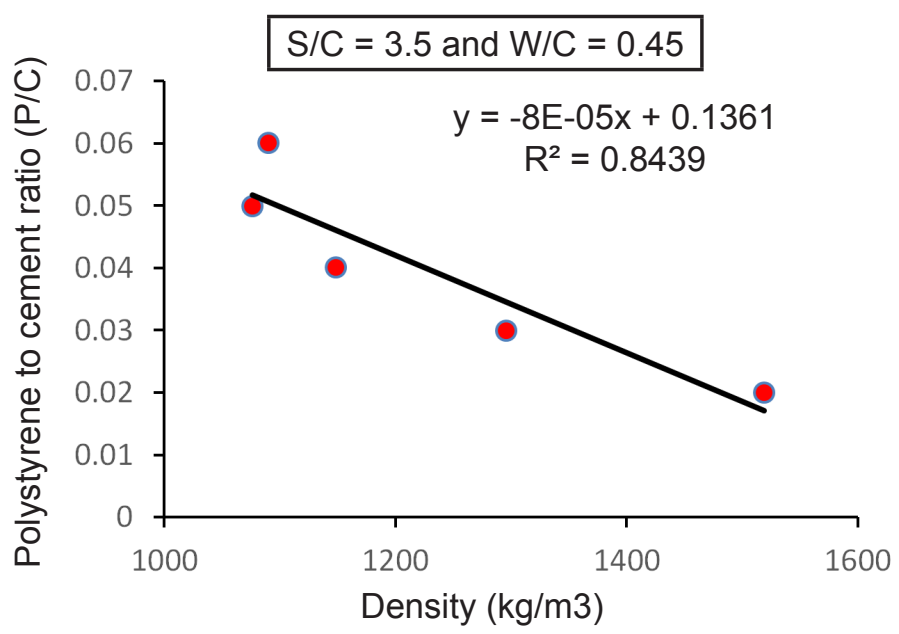

(i)

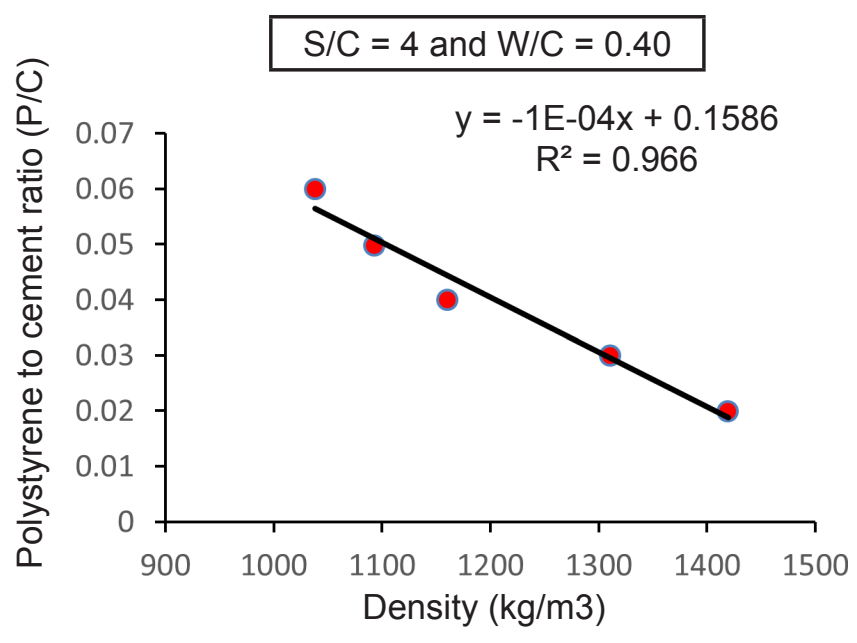

(k)

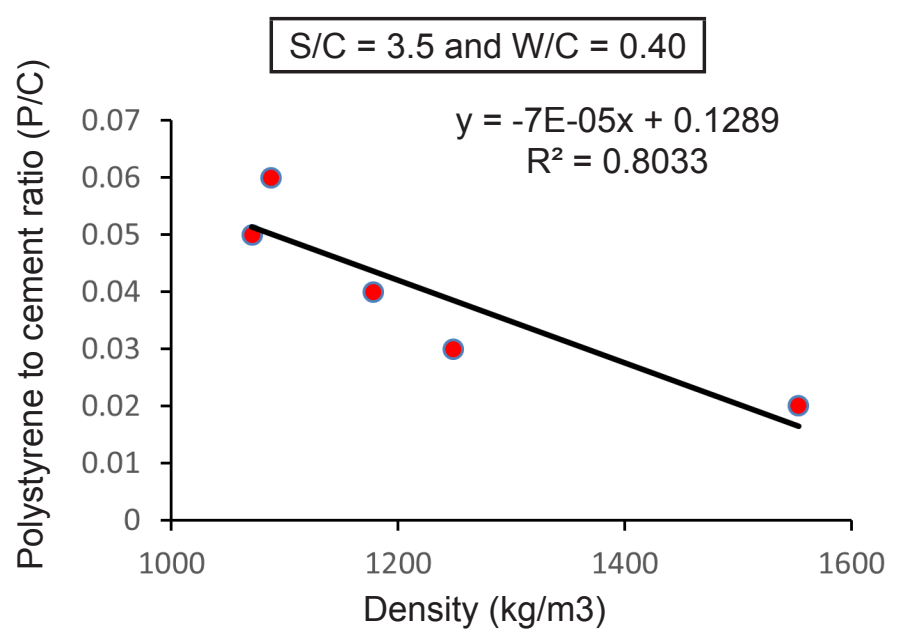

(h)

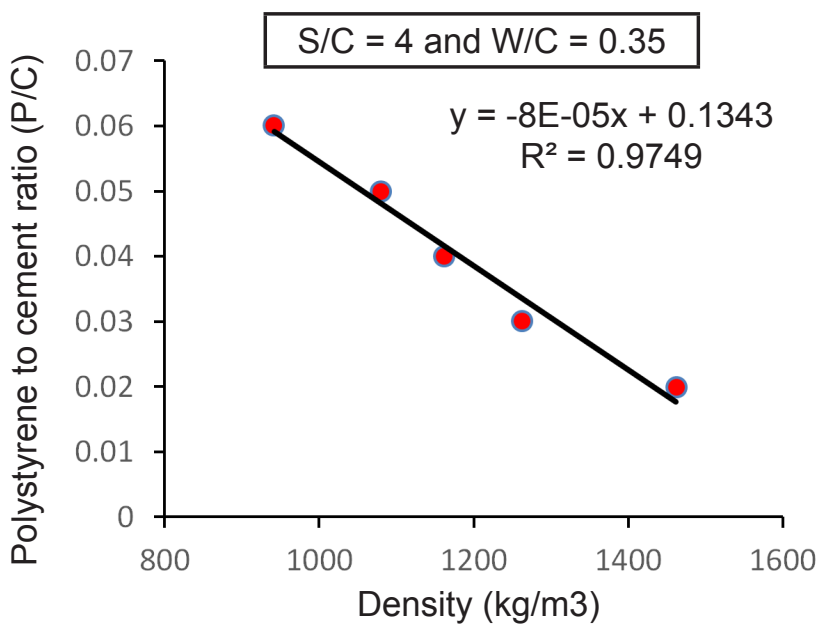

(j)

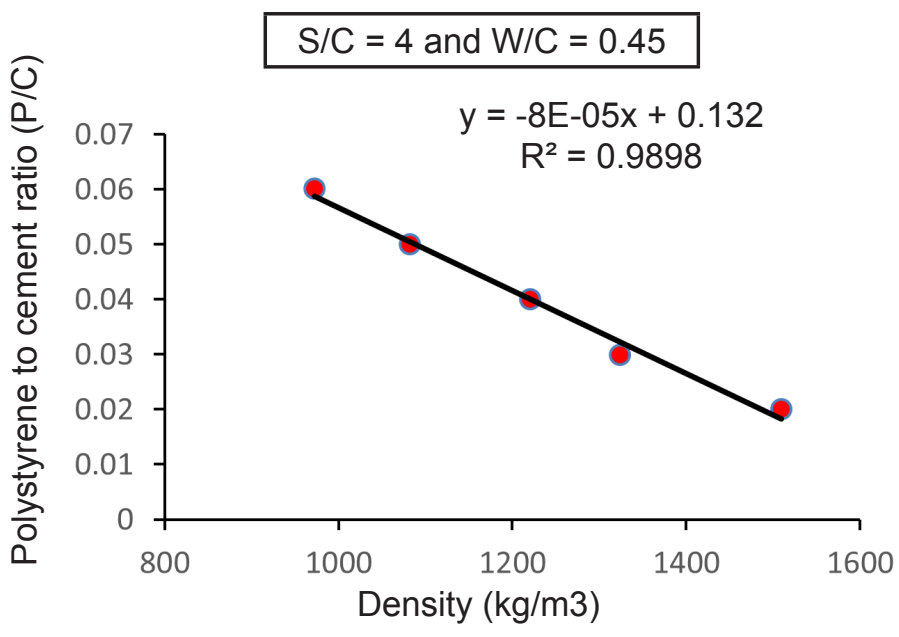

(l)

Figure 8: the relationships between dry density and $(P / C)$ ratios

\section{CONCLUSIONS}

This paper shows test results of polystyrene lightweight concrete having different density, compressive strength and mix proportions. Some conclusions can be drawn as follows:
1. The compressive strength decreases as the $(P / C)$ ratio increases. For all mixes the maximum decrease occurred when the $(P / C)$ ratio was increased from 0.02 to 0.03 . 
2. Compressive strength is more sensitive to the polystyrene content than dry density as the reduction in compressive strength reached about $82 \%$ in comparison with $36 \%$ for dry density when the $(\mathrm{P} / \mathrm{C})$ ratio was increased from 0.02 to 0.06 .

3. Some mixtures showed an increase in the compressive strength as the $(\mathrm{W} / \mathrm{C})$ ratio was increased and this may be attributed to the good compaction achieved in these mixes.

4. Considerably high relationships were obtained between compressive strength and $(\mathrm{P} / \mathrm{C})$ ratio as the regression analysis provided an average correlation coefficient $\left(R^{2}\right)$ of 0.9751 , so, they can be of use in determining mix proportions of polystyrene lightweight concrete based on the required compressive strength.

5. Dry density increases with the increase of $(S / C)$ ratio and decreases of $(\mathrm{P} / \mathrm{C})$ ratio in the mixture. $A$ consideable decrease in the dry density values was observed as the $(\mathrm{P} / \mathrm{C})$ ratio in the mixture was increased from 0.02 to 0.06 .

6. A Considerably high relationships were obtained between dry densities and $(P / C)$ ratio of the polystyrene lightweight concrete as the regression analysis provided an average correlation coefficient $\left(R^{2}\right)$ of 0.9401 , so, they can be of use to determine the proportion of the mixtures based on the required dry density.

\section{REFERENCES}

1. Neville, A.M. (1981). Properties of Concrete. The Pitman Press, London.

2. ACl Committee 213R-87 (1987). Guide for Structural Lightweight Aggregate Concrete. ACI Manual of Concrete Practice, Part 1, American Concrete Institute, Farmington Hills.

3. Kohling, K. (1960). The manufacture of lightweight concrete using pre-expanded styropore particles as aggregates. betorstein - zeitung, Vol. 26, pp. 208 $-212$.

4. Maura, G. (1978). Light weight concrete made with expanded substituted polystyrene. IL Cement (Rome), Vol. 75, part 1, pp. $21-29$.

5. Parton, G. M., and Shendy-EL- Barbary, M.E. (1982). Poly styrene - beads concrete properties and mix design. Journal of cement composite and light weight concrete, Vol. 4, No 3, pp. 153 - 161.

6. Sabaa, B., and Ravindrarajah, R.S. (1997). Engineering Properties of Lightweight Concrete Containing Crushed Expanded Polystyrene Waste. Materials Research Society, 1997 Fall Meeting Symposium MM: Advances in Materials for Cementitious Composites December 1-3, Boston, USA
7. Kuhail, Z., and Shihada, S. (2003). Mechanical properties of polystyrene lightweight concrete. Journal of the Islamic University of Gaza, Vol. 11, No. 2, pp. 93-114.

8. Kan, A, and Demirboga, R. (2007). Effect of cement and EPS beads ratios on compressive strength and density of lightweight concrete. Indian Journal of Engineering \& Materials Sciences, Vol. 14, pp. 158-162

9. Herki, B.A., Safary, Z., and Khalid, O. (2016). Engineering Properties of Sustainable Lightweight Concrete Using Waste Polystyrene. International Journal of Engineering and Innovative Technology (IJEIT) Volume 5, Issue 12

10. Abd, S. M., Dhamya G., Hattem, M., and Khalil, D. (2016). Effective Replacement of Fine Aggregates by Expanded Polystyrene Beads in Concrete. International Journal of Engineering Research and Science \& Technology (IJERST), Vol. 5, No. 3.

11. Duc Hoang Minh, D. H., and Ly Le Phuong, L. L. (2018). Effect of matrix particle size on EPS lightweight concrete properties. MATEC Web of Conferences 251, 01027 (2018), IPICSE-2018 https://doi. org/10.1051/matecconf/201825101027

12. Bedekovic, G., Grcic, I., Vucinic, A. A., and Premur, V. (2019). Recovery of waste expanded polystyrene in lightweight concrete production. The Mining-Geology-Petroleum Engineering Bulletin, UDC: 624.01, DOI: 10.17794/rgn.2019.3.8

13. Ubi, Stanley E., Okafor, F. O., and Mama, B. O., (2020). Optimization of Compressive Strength of Polystyrene Lightweight Concrete Using Scheffe"s Pseudo and Component Proportion Models. SSRG International Journal of Civil Engineering (SSRG-IJCE), Volume 7, Issue 6, June.

14. ASTM, "Specification for Portland cement," ASTM C 150, ASTM, West Conshohocken, Pa, USA, 2007.

15. British Standards Institution, B.S. 882: 1992. Aggregate from natural sources for concrete, London

16. British Standards Institution, B.S.1881, Part 116, 1989, Method for Determination of Compressive Strength of Concrete Cubes, British Standard Institute London.

17. ASTM C 642, "Standard Test Method for Density, Absorption, and Voids in Hardened Concrete", Annual Book of ASTM, Standards, Vol. 04.02, 1997.

Paper submitted: 10.07.2020.

Paper accepted: 20.10.2020.

This is an open access article distributed under the CC BY 4.0 terms and conditions. 